\title{
IDENTIFICATION OF FREQUENT PROMOTER METHYLATION OF DEATH-ASSOCIATED PROTEIN KINASE IN LIQUID-BASED PAPANICOLAOUS TEST SAMPLES IN VIETNAMESE POPULATION
}

\author{
PHUONG KIM TRUONG ${ }^{1,2}$, THUAN DUC LAO ${ }^{1,2}$, THUY AI HUYEN LE ${ }^{2 *}$ \\ ${ }^{1}$ Department of Molecular and Environmental Biotechnology, Faculty of Biology and Biotechnology, University of Science, Vietnam \\ National University Ho Chi Minh City, Vietnam. ${ }^{2}$ Department of Pharmaceutical Biotechnology, Faculty of Biotechnology, Ho Chi Minh City \\ Open University, Ho Chi Minh City, Vietnam. Email: thuy.lha@ou.edu.vn
}

Received: 01 May 2017, Revised and Accepted: 27 June 2017

\section{ABSTRACT}

Objective: The infection of high-risk human papillomavirus (HPV) genotypes, particularly HPV-16 and HPV-18, is known to cause cervical cancer (CC); however, aberrant DNA methylation of death-associated protein kinase (DAPK), a member of tumor suppressor gene family, are required for cervical tumorigenesis. The aim of our study was to evaluate the hypermethylation frequency of CpG belonged to DAPK promoter, in Vietnamese patients, as well as to study about the association between hypermethylation, and high-risk HPV infection leading to CC.

Methods: Methylation-specific-polymerase chain reaction (MSP) was performed to analyze methylation status from 109 liquid-based papanicolaous test samples, collected from local hospital and were identified whether HPV/or non-HPV, high-risk/low-risk HPV infection, then was confirmed by sequencing.

Results: In the case of high-risk HPV infection, the frequency of DAPK gene hypermethylation was $66.67 \%$ (24 of 36 cases). Meanwhile, low hypermethylation status was found in low-risk and non-HPV infection, counting for $12.0 \%$ (3 of 25 cases), $2.1 \%$ ( 1 of 48 cases), respectively. Significant association of DAPK hypermethylation with high-risk, low-risk, and non-HPV infection was observed (p<0.0001). The DAPK hypermethylation increased the possibility to CC in the case of high-risk HPV infected with high incidence: Odds ratio=34.5 (95\% confidence interval [CI] $=10.15-117.23$, $\mathrm{p}<0.01)$, relative risk $=12.2(95 \% \mathrm{CI}=4.56-32.42, \mathrm{p}<0.01)$.

Conclusion: Based on those data, it suggested that MSP carried out on noninvasive samples will lead to potential method to screening, diagnosis and early diagnosis of cervical carcinoma in Vietnamese population.

Keywords: Cervical cancer, Hypermethylation, Methylation-specific-polymerase chain reaction, Death-associated protein kinase, Vietnamese population.

(C) 2017 The Authors. Published by Innovare Academic Sciences Pvt Ltd. This is an open access article under the CC BY license (http://creativecommons. org/licenses/by/4. 0/) DOI: http://dx.doi.org/10.22159/ajpcr.2017.v10i10.19528

\section{INTRODUCTION}

Persistent infection with high-risk genotype of human papillomavirus (HPV) has been known to be associated with the cervical carcinogenesis [1-4]. Among high-risk HPV genotypes, HPV-16 and18 , which is identified as the common high-risk HPV genotypes and being the key role for the majority of cervical cancer (CC), counting for approximately $70.0 \%$ [5-9]. In Vietnam, the prevalence of cervical infection with HPV-16 and/or-18 among women was ranged from $3.1 \%$ to $7.4 \%$ [10]. Notably, the infection of high-risk HPV genotypes is considered as not exclusive prerequisites for cervical carcinogenesis [11]. Additional genetic and epigenetic alterations are required for progression from precancerous disease to invasive cancer $[8,12,13]$. Among the putative molecular alterations involved in cervical carcinogenesis process, promoter hypermethylation, an important epigenetic modification, has long been found to be a major mechanism of inactivation of tumor suppressor genes (TSGs), which has been considered as an early epigenetic event in driving carcinogenesis of many human cancers, including cancer of cervix [14-16].

The death-associated protein kinase (DAPK), a member of TSG family, located at 9q21.33, was a calcium/calmodulin-dependent seurine/threonine kinase involved in multiple cellular signaling pathways that trigger cell survival, apoptosis, and autophagy [14-18]. DAPK has been proved as participating in interferon- $\gamma$, Fas, tumor necrosis factor- $\beta$, extracellular signal-regulated kinase, C-myc and E2F-induced apoptosis, which suggested that DAPK is a conjunction of all kinds of apoptosis signals $[18,19]$. DAPK promoter aberrant hypermethylation has been reported involving in many primary tumors, including cervical carcinoma $[16,17]$. In Vietnam, the studies revealed to evaluation of TSGs had been reported, but still limited. Given examples, in our previous studies, the significant association between the hypermethylation of many TSGs, including p 16 INK $4 \alpha$, BRCA1, Cyclin D2, GSTP1, and RASSF1A, and breast cancer were reported. However, notably, there is no data regarding DAPK promoter hypermethylation in Vietnamese, which is considered involving CC [20-22]. Therefore, the aim at this study is to evaluate the frequency of hypermethylation of $\mathrm{CpG}$ which is belonged to promoter of DAPK gene, in Vietnamese population, as well as, to study about the association between the epigenetic event, hypermethylation, and high-risk HPV infection leading to the cancer of cervix. It was also noted here is the usage of liquid-based papanicolaous test specimens (Pap), noninvasive materials, to develop noninvasive method for prognosis and early diagnosis of CC in Vietnamese patients based on DNA methylationspecific-polymerase chain reaction (MSP).

\section{METHODS}

Ethics statement

We used anonymized routine specimen surplus to clinical requirements for assay validation, adhering to a governance framework agreed by and with a Medic Medical Center and Au Lac Clinic Laboratory ethics agreement relating to the use of specimen surplus to clinical needs. 
Sample collection

A total of 109 liquid-based Pap test samples were archived and admitted from the Au Lac Clinic, Vietnam. For input confirmed, the detection of HPV was carried out using LightPoweriVA HPV genotype PCR-reverse dot blot Kit (Code: VA.A02-003E, Viet-A Corporation, Vietnam). As the results, all samples were divided into two groups: Negative HPV infection group, which consisted of 48 samples; and positive HPV infection group, in which composed of 36 high-risk HPV (HPV genotype 16,18 and other high-risk genotypes) infected samples and 25 low-risk HPV infected samples.

\section{DNA isolation, bisulfite modification, MSP assay}

A total of genomic DNA was isolated from Pap samples by phenol/ chloroform method. Then, DNA concentration of DNA was quantified by the absorbance at $\mathrm{OD}_{260}$ and $\mathrm{OD}_{280^{\circ}}$ The pure preparation of DNA with $\mathrm{OD}_{260} / \mathrm{OD}_{280}$ ratio values of $1.8-2.0$ was used to the bisulfite DNA modification assay. The bisulfite modification was carried out with approximately $2 \mu$ g genomic DNA of each sample by DNA modification Kit (Epitect Kit, Qiagen). The final precipitate was eluted in a volume of $20 \mu \mathrm{l}$ for MSP assay.

MSP assay was carried out in a total of $15 \mu \mathrm{l}$ containing $3 \mu \mathrm{l}$ bisulfitemodified template DNA, 0.75 unit iTaq DNA polymerase (Biorad). MSP reaction was subjected to initial incubation at $95^{\circ} \mathrm{C}$ for 5 minutes, followed by 40 cycles at $95^{\circ} \mathrm{C}$ for $30 \mathrm{~s}, \times^{\circ} \mathrm{C}$ for $30 \mathrm{~s}, 72^{\circ} \mathrm{C}$ for $30 \mathrm{~s}$, and $72^{\circ} \mathrm{C}$ for 6 minutes for final incubation. (Note: $\mathrm{X}$ was the annealing temperature of each specific methylated or unmethylated primer to candidate gene). The sequences of primers and $x^{\circ} \mathrm{C}$ for each primer annealing were noted in Table 1. Each PCR product was directly loaded onto a $2.0 \%$ agarose gel, stained with ethidium bromide, and directly visualized under ultraviolet illumination. Then, MSP products were sequencing to confirm the specificity of primers, examine the efficiency of bisulfite modification, and the hypermethylation status of target gene.

\section{Statistical analysis}

Statistical analyses were performed using Medcalc $^{\circledR}$ Version 12.7.0.0 that used the Chi-square test for sample size. The correlation between methylation status and HPV infected status was examined using the Chi-squared test. The differences of methylation frequencies of DAPK among groups were considered statistically significant for $\mathrm{p} \leq 0.05$. Moreover, the odds ratio (OR), relative risk (RR) with $95 \%$ confidence intervals $(\mathrm{CI})$ were also evaluated.

\section{RESULTS}

Hypermethylation status of DAPK promoter

In this study, MSP method was applied in DAPK promoter methylation detection. According to Table 2, in general, it indicated that, in the group of high-risk HPV-infection, the methylation frequency was $66.67 \%(24$ of 46 cases) as significant higher than in two other groups, which were $12.0 \%$ ( 3 of 25 cases) and $2.08 \%$ ( 1 of 48 cases) for low-risk HPVinfected group and non-HPV infected group, respectively. Moreover, in high-risk HPV infected samples, the methylation frequency, counting for $66.67 \%$, was higher than unmethylation frequency counting for $33.33 \%$. Conversely, the status of unmethylation in low-risk HPV or non-HPV infected group was higher than the methylation in both lowrisk HPV infected group and non-HPV-infected group. Comparison with previous studies, giving example, the frequency of DAPK methylation

Table 1: Primer sequences were used in this study

\begin{tabular}{|c|c|c|c|}
\hline Primer name & Primer sequence $\left(5^{\prime}-3^{\prime}\right)$ & $\mathrm{X}^{\circ} \mathrm{C}$ & $\mathbf{P}$ \\
\hline DAPK-MF & 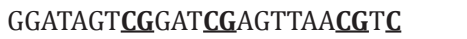 & 57 & 172 \\
\hline DAPK-MR & CATAAACGCCAACGCCGAAAA & & \\
\hline DAPK-UF & GGAGGATAGTTGGATTGAGTTAATGTT & 53 & 176 \\
\hline DAPK-UF & 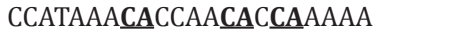 & & \\
\hline
\end{tabular}

Note:CpG islands were bold and underlined, $\mathrm{X}^{\circ} \mathrm{C}$ : Primer annealing

temperature, M: Methylated, U: Unmethylated, F: Forward, R: Reverse, P:

Product size, DAPK: Death-associated protein kinase was rather higher than Leung et al. (2008), which was 56.08\%, carried on cervical samples. Moreover, the MSP product of DAPK was also observed in the band of both 172, 176 lengths in case of methylation and unmethylation, respectively, shown in Fig. 1. Then, the results of MSP were confirmed by sequencing and shown in Fig. 2. Concerning to the analysis of MSP product, shown in Figs. 1 and 2, we found that there was only one band with the length of 172 bps (in the case of methylated samples), and 176 bps (in the case of unmethylated samples). Then, MSP product was confirmed by MSP sequencing, according to Fig. 2, we successfully carried the bisulfite modification and MSP assay. By sequencing, making the comparison between the non-bisulfite modified (Fig. 2a) and bisulfite modified (Fig. 2b), all methylated cytosines were unchanged, which were marked as green characters. Otherwise, all the unmethylated cytosines were totally changed into thymine in bisulfite sequence. In addition, four methylated CpG sites were observed in methylated reverse primer, which was according to the primer designed. As shown in Fig. 2c and d, the signals of peaks in MSP product sequencing were quite good for reading nucleotide sequencing. Therefore, for those reasons, it was concluded that the bisulfite modification was successfully carried out.

\section{Calculation of OR and RR}

In this study, through the analysis of methylation or unmethylation status of DAPK, OR, RR was also calculated. The OR and RR were evaluation between high-risk HPV infection group and low-risk HPV group combined with non-HPV group, the OR and RR were 34.5 (95\% CI=10.15-117.23, $\mathrm{p}<0.01), 12.2$ (95\% $\mathrm{CI}=4.56-32.42, \mathrm{p}<0.01)$, respectively.

\section{DISCUSSION}

In addition to HPV infection, particularly high-risk HPV genotypes, recently, the inactivation of TSGs due to promoter hypermethylation has been reported that it is a frequent mechanism plays an important criterion in cervical carcinogenesis [8,14-16]. In this study, we demonstrated that the hypermethylation of the DAPKgene promoter was found in high frequency in high-risk HPV infected samples, counting for $66.67 \%$ (24 of 36 samples), compared to low-risk HPV infected samples, counting for $12.0 \%$ (3 of 25 samples) and non-HPV infected samples, counting for $2.08 \%$ (1of 48 samples). In the previous study, DAPK, a proapoptotic serine/threonine kinase, has been postulated to function as TSGs, plays a role in tumor pathogenesis and metastasis $[18,19]$. The inactivation of DAPK, mainly by promoter hypermethylation, has been reported in CC. Notably, promoter hypermethylation of DAPK was investigated and found to be significant higher in high-risk HPV infected samples than low-risk HPV infected samples and non-HPV infected samples $(\mathrm{p}<0.0001)$. It is thought that some Oncogenic viruses are known to interact with, and to modulate the expression of DNA methyltransferases (DNMTs), resulting to in the transactivation and transrepression of both cellular and viral genes $[23,24]$. Even though the relationship between HPV and aberrant methylation in CC is not well understood, some reports suggested E6 and E7 oncoprotein of high-risk HPV increase the expression and activity of DNMT1, which is the major mammalian enzyme responsible for maintaining CpG methylation patterns in the cell following replication, through the suppression of TSG p53 [25,26]. In addition, HPV-16 E7 bind to DNMT1 and precipitate, upregulate DNA methyltransferase activity, increased the hypermethylation in many TSGs led to the development

$200 \mathrm{bp} \quad \frac{(1)}{\mathrm{MW} \mathrm{U}} \frac{(2)}{\mathrm{MU}} \frac{(3)}{\mathrm{MU}} \frac{(4)}{\mathrm{MU}} \frac{(5)}{\mathrm{MU}} \frac{(6)}{\mathrm{MU}} \frac{(7)}{\mathrm{MU}} \frac{(8)}{\mathrm{MU}} \frac{(9)}{\mathrm{MU}} \mathrm{NC}$

Fig. 1: Methylated promoter of death-associated protein kinase gene was analyzed on some clinical samples by methylationspecific-polymerase chain reaction (MSP). The MSP product was $172 / 176$ bps in length. (1 and 2 ) low-risk human papillomavirus (HPV) infected; (3-5): Non-HPV infected, (6-9): High-risk HPV infected samples, MW: Molecular weight marker, 100 bp; NC: Negative control 


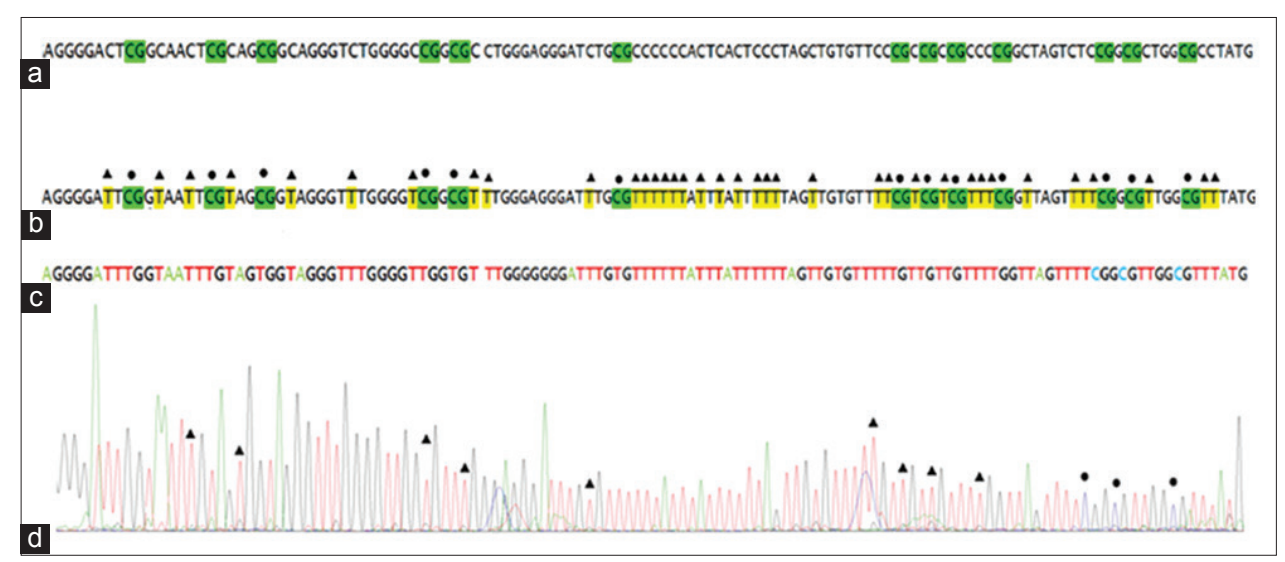

Fig. 2: Sequencing profile of segment methylated of death-associated protein kinase. CpG sites were in the green highlight; cytosine did not depend on the CpG site were in yellow. (a) DNA sequence was without bisulfite modified, (b) DNA sequence was bisulfite modified, (c and d) The death-associated protein kinase sequencing using the forward primer

Table 2: The methylation profile for DAPK gene

\begin{tabular}{lll}
\hline Samples & DAPK n (\%) & \\
\cline { 2 - 3 } & $\mathbf{M}$ & $\mathbf{U}$ \\
\hline High-risk HPV infected & $24(66.67)$ & $12(33.33)$ \\
Low-risk HPV infected & $3(12.0)$ & $22(88.0)$ \\
Non-HPV infected & $1(2.08)$ & $47(97.72)$ \\
p value & $<0.0001$ & \\
\hline
\end{tabular}

M: Methylated, U: Unmethylated, DAPK: Death-associated protein kinase,

HPV: Human papillomavirus

of human cancer of cervix [22]. In this study, the hypermethylation of DAPK promoter was detectable in liquid-based Pap test sample by MSP method. The advantage of using Pap test samples is one kind of noninvasive samples, contains exfoliating cells from the transformation zone of the cervix; thus, the presence of DNA in exfoliating cells has offered an opportunity to find out the predictive and diagnosis biomarkers for CC [27-29]. In addition, MSP method is the simple, rapid and inexpensive method that determine the methylation status of CpG islands. Extraordinary the sensitivity of $0.1 \%$ can be achieved in MSP with a low false positive rate [18,30]. Moreover, MSP method shows an advantage in the requirement of only small quantities of DNA and could be applied on DNA extracted from noninvasive samples [30].

The hypermethylation of DAPK in cervical patients was significantly associated with an approximately 34.5 -fold increase in the highrisk HPV infected group than compared to low-risk and/or non-HPV infected group $(\mathrm{OR}=34.5,95 \% \mathrm{CI}=10.15-117.23, \mathrm{p}<0.01)$. Concerning to RR value, it indicated that high-risk HPV infection significantly increased 12.2 times in the risk of DAPK hypermethylation, leading to the inactivation of DAPK (RR=3.31,95\% CI=1.75-6.26, $\mathrm{p}<0.01$ ), compared to without DAPK promoter hypermethylation.

These observations open up to the possibility that the association between the DAPK promoter hypermethylation and high-risk HPV infection will offer the potential method, which based on MSP method carried out in noninvasive samples, for screening, predictive and diagnosis biomarkers for human CC in Vietnamese population.

\section{CONCLUSION}

In summary, hypermethylation of $\mathrm{CpG}$ islands within the promoter region of DAPK, which frequency was counting for $66.67 \%, 12.0 \%$, $2.1 \%$ for high-risk, low-risk and non-HPV infection, respectively, is a feature of human cancer of cervix, involving in the HPV infection in Vietnamese population. Moreover, the OR and RR were found in the high value, counting for 34.5 and 12.2 , respectively. Consequently, we propose that the screening, which based on the combination of both high-risk HPV detection and DAPK gene's promoter methylation, will be an auspicious characteristic for early predictive and diagnosis of CC.

\section{ACKNOWLEDGMENTS}

We wish to express our thanks the research project sponsored by Ho Chi Minh City Open University, Ho Chi Minh City, Vietnam. We thank all the recruited participants in this work and all the staff members of Medic Medical Center and Au Lac Clinic Laboratory, for collecting the samples used in these studies.

\section{REFERENCES}

1. Kirti, Prabhakar PK. Human papilloma virus associated CC: A review. Asian J Pharm Clin Res 2016;9:14-7.

2. Malpica A, Matisic JP, Niekirk DV, Crum CP, Staerkel GA, Yamal JM, et al. Kappa statistics to measure inter-rater and intra-rater agreement for 1790 cervical biopsy specimens among twelve pathologists: Qualitative histopathologic analysis and methodologic issues. Gynecol Oncol 2005;993 Suppl 1:S38-52.

3. Jmaah AK, Malla S, Kumar RS. Cloning and characterization of high risk human papilloma virus (HPV). Asian J Pharm Clin Res 2014;7 Suppl 2:61-5.

4. Gupta M, Dahiya J, Marwaha RK, Dureja H. Therapies in cancer treatment: An over view. Int J Pharm Pharm Sci 2015;7:1-9.

5. zur Hausen H. Papillomavirus infections-a major cause of human cancers. Biochim Biophys Acta 1996;1288(2):F55-78.

6. Burd EM. Human papillomavirus and cervical cancer. Clin Microbiol Rev 2003;16(1):1-17.

7. Jenkins D. A review of cross-protection against oncogenic HPV by an HPV-16/18 AS04-adjuvanted cervical cancer vaccine: Importance of virological and clinical endpoints and implications for mass vaccination in cervical cancer prevention. Gynecol Oncol 2008;110:S18-25.

8. Yang HJ. Aberrant DNA methylation in cervical carcinogenesis. Chin J Cancer 2013;32(1):42-8.

9. Castle PE, Maza M. Prophylactic HPV vaccination: Past, present, and future. Epidemiol Infect 2016;2:1-20.

10. Vu LT, Bui D, Le HT. Prevalence of cervical infection with HPV type 16 and 18 in Vietnam: Implications for vaccine campaign. BMC Cancer 2013;13:53

11. Lazo PA. The molecular genetics of cervical carcinoma. Br J Cancer 1999;80(12):2008-18.

12. Nath A, Priyanka KK, Anshu AK, Singh CK, Behera S, Singh JK. Hypomethylation of deoxyribonucleic acid in testicular tissue due to arsenic exposure in mice. Asian J Pharm Clin Res 2006;9:294-6.

13. Sherzay N, Chitakar E. Epigenetics: Effect of environmental factors on human genome. Int J Pharm Pharm Sci 2016;8:1-6.

14. Baylin SB, Esteller M, Rountree MR, Bachman KE, Schuebel K, Herman JG. Aberrant patterns of DNA methylation, chromatin formation and gene expression in cancer. Hum Mol Genet 2001;10(7):687-92.

15. Lu Q, Ma D, Zhao S. DNA methylation changes in cervical cancers. Methods Mol Biol 2012;863:155-76.

16. Xiong J, Li Y, Huang K, Lu M, Shi H, Ma L, et al. Association between 
DAPK1 promoter methylation and cervical cancer: A meta-analysis. PLoS One 2014;9(9):e107272.

17. Leung RC, Liu SS, Chan KY, Tam KF, Chan KL, Wong LC, et al. Promoter methylation of death-associated protein kinase and its role in irradiation response in cervical cancer. Oncol Rep 2008;19(5):1339-45

18. Zhao XL, Meng ZY, Qiao YH, Zhang HL. Promoter methylation of DAPK gene in cervical carcinoma. Ai Zheng 2008;27(9):919-23.

19. Anjum R, Roux PP, Ballif BA, Gygi SP, Blenis J. The tumor suppressor DAP kinase is a target of RSK-mediated survival signaling. Curr Biol 2005;15(19):1762-7.

20. Truong PK, Lao TD, Doan TP, Le TA. BRCA1 promoter hypermethylation signature for early detection of breast cancer in the Vietnamese population. Asian Pac J Cancer Prev 2014;15:9607-10.

21. Truong PK, Lao TD, Doan TP, Le TA. Evaluation of aberrant p16INK4a promoter $\mathrm{CpG}$ methylation and its application in Vietnamese breast cancer patients. In: Proceedings of the Fourth International Conference on Advances in Applied Science and Environmental Engineering; 2015. p. 46-50.

22. Truong PK, Lao TD, Doan TP, Le TA. Loss of expression of cyclin d2 by aberrant DNA methylation: A potential biomarker in vietnamese breast cancer patients. Asian Pac J Cancer Prev 2015;16(6):2209-13.

23. Whiteside MA, Siegel EM, Unger ER. Human papillomavirus and molecular considerations for cancer risk. Cancer 2008;113 10 Suppl:2981-94.

24. Leonard SM, Wei W, Collins SI, Pereira M, Diyaf A, ConstandinouWilliams $\mathrm{C}$, et al. Oncogenic human papillomavirus imposes an instructive pattern of DNA methylation changes which parallel the natural history of cervical HPV infection in young women. Carcinogenesis 2012;33(7):1286-93.

25. Burgers WA, Blanchon L, Pradhan S, de Launoit Y, Kouzarides T, Fuks F. Viral oncoproteins target the DNA methyltransferases. Oncogene 2007;26(11):1650-5.

26. Au Yeung CL, Tsang WP, Tsang TY, Co NN, Yau PL, Kwok TT. HPV-16 E6 upregulation of DNMT1 through repression of tumor suppressor p53. Oncol Rep 2010;24(6):1599-604.

27. Kahn SL, Ronnett BM, Gravitt PE, Gustafson KS. Quantitative methylation-specific PCR for the detection of aberrant DNA methylation in liquid-based Pap tests. Cancer 2008;114(1):57-64.

28. Qureshi SA, Bashir MU, Yaqinuddin A. Utility of DNA methylation markers for diagnosing cancer. Int J Surg 2010;8(36):194-8.

29. Fleischhacker M, Schmidt B. Circulating nucleic acids (CNAs) and cancer - A survey. Biochim Biophys Acta 2007;1775(1):181-232.

30. Herman JG, Graff JR, Myöhänen S, Nelkin BD, Baylin SB. Methylationspecific PCR: A novel PCR assay for methylation status of $\mathrm{CpG}$ islands. Proc Natl Acad Sci U S A 1996;93(18):9821-6. 